\title{
Adaptação transcultural do Questionário ABILHAND específico para indivíduos pós-acidente vascular encefálico
}

\section{Cross-cultural adaptation of the ABILHAND Questionaire, specific for individuals after stroke}

\author{
Marluce Lopes Basílio ${ }^{1}$, Iza de Faria-Fortini ${ }^{2}$, Fernanda Sabine Nunes \\ Assumpção ${ }^{3}$, Augusto Cesinando de Carvalho ${ }^{4}$, Luci Fuscaldi Teixeira-Salmela ${ }^{5}$
}

http://dx.doi.org/10.11606/issn.2238-6149.v28i1p19-26

\begin{abstract}
Basílio ML, Faria-Fortini I, Assumpção FSN, Carvalho AC, Teixeira-Salmela LF. Adaptação transcultural do Questionário ABILHAND específico para indivíduos pós-acidente vascular encefálico. Rev Ter Ocup Univ São Paulo. 2017 jan.-abr.;28(1):19-26.
\end{abstract}

RESUMO: Introdução: O ABILHAND, específico para indivíduos pós-Acidente Vascular Encefálico (AVE), é um questionário para avaliação de habilidade manual e foi construído baseado no modelo Rasch de mensuração, que permite converter escores ordinais em medidas lineares. Objetivo: Adaptar transculturalmente o ABILHAND para uso no Brasil. Método: $\mathrm{O}$ processo de adaptação transcultural seguiu procedimentos padronizados: tradução inicial, síntese das traduções, retrotradução, comitê de especialistas e teste da versão pré-final, para o qual foram recrutados 10 indivíduos. Resultados: A comparação entre a versão original e a retrotraduzida demonstrou boa equivalência entre as versões. O comitê de especialistas adequou a descrição dos itens às regras gramaticais da língua portuguesa e acrescentou uma expressão em um item, para melhorar a equivalência conceitual. No teste da versão pré-final, não foram observadas dificuldades de compreensão durante a aplicação do questionário. Conclusão: O ABILHAND-Brasil apresentou satisfatório grau de equivalência semântica, idiomática, cultural e conceitual. Os resultados desse estudo viabilizam o uso do ABILHAND no Brasil, que pode se constituir em uma boa opção para se avaliar o desempenho de indivíduos pós-AVE em atividades manuais.

DESCRITORES: Acidente vascular cerebral, Questionário, Extremidade superior, Atividades cotidianas.
Basílio ML, Faria-Fortini I, Assumpção FSN, Carvalho AC, Teixeira-Salmela LF. Cross-cultural adaptation of the ABILHAND Questionaire, specific for individuals after stroke. Rev Ter Ocup Univ São Paulo. 2017 Jan.-Apr.;28(1):19-26.

ABSTRACT: Introduction: The ABILHAND, specific for individuals after stroke, is a questionnaire for the assessment of manual ability and was developed based upon the Rasch measurement model, which allows for the conversion of ordinal scores into linear measures. Objective: To cross-culturally adapt the ABILHAND for its use in Brazil. Method: The process of crosscultural adaptation followed standardized guidelines: translation, back-translation, summary of the translations, expert committee evaluation, and testing of the pre-final version, for which 10 individuals were recruited. Results: The comparison between the original and the translated versions showed good equivalence between the versions. The expert committee adapted the description of the items to the grammatical rules of the Portuguese language and added an expression in an item, to improve conceptual equivalence. During the pre-final version testing, no comprehension difficulties were observed during the application of the questionnaire. Conclusion: The ABILHAND-Brazil showed satisfactory semantic, linguistic, cultural, and conceptual equivalence. These results supported the use of the ABILHAND in Brazil, which can be a good option for the evaluation of the performance of post-stroke individuals in manual activities.

KEYWORDS: Stroke; Questionnaire, Upper extremity, Activities of daily living.

Este trabalho é parte da dissertação de mestrado ‘Adaptação transcultural e propriedades de medida do ABILHAND-Brasil: uma medida de habilidade manual para indivíduos com hemiparesia’, de Marluce Lopes Basílio, Programa de Pós-Graduação em Ciências da Reabilitação - Universidade Federal de Minas Gerais.

Agências de fomento: $\mathrm{CNPq}$, FAPEMIG

1. Programa de Pós-graduação em Ciências da Reabilitação, Universidade Federal de Minas Gerais (UFMG), Belo Horizonte, MG. E-mail: marlucelb@yahoo.com.br

2. Hospital das Clínicas, UFMG/Filial da Empresa Brasileira de Serviços Hospitalares (EBSERH), Belo Horizonte, MG. Email: izafaria@yahoo.com.br

3. Centro de Reabilitação Noroeste da Rede do Sistema Único de Saúde, Belo Horizonte, MG. Email: fernandasabine@yahoo.com.br

4. Departamento de Fisioterapia, Universidade Estadual Paulista Júlio de Mesquita Filho (UNESP) - Campus Presidente Prudente, SP, Brasil. Email: augustocesinando@gmail.com

5. Departamento de Fisioterapia, UFMG, Belo Horizonte, MG. Email: 1fts@ufmg.br

Endereço para correspondência. Marluce Lopes Basílio. Departamento de Fisioterapia, Universidade Federal de Minas Gerais. Avenida Antônio Carlos, 6627, Campus Pampulha. 31270-901 Belo Horizonte, MG, Brasil. E-mail: marlucelb@yahoo.com.br 


\section{INTRODUÇÃO}

$\mathrm{O}$ acidente vascular encefálico (AVE) é um dos principais problemas de saúde no Brasil. Após um AVE, há uma série de consequências funcionais, que podem resultar em incapacidades e redução da qualidade de $v i^{2}{ }^{2}$. Em indivíduos pós-AVE, as consequências funcionais relacionadas aos membros superiores (MMSS) estão entre as mais relevantes ${ }^{3}$, uma vez que os MMSS contribuem de maneira significativa para a realização de várias atividades de vida diária, consideradas essenciais para uma vida independente ${ }^{4}$. Diante disso, na reabilitação de pessoas acometidas por um AVE, restaurar a funcionalidade referente ao uso dos MMSS é um objetivo primordial. Consequentemente, a avaliação funcional dos MMSS é fundamental, tanto na prática clínica quanto na pesquisa, para determinar a efetividade de intervenções e monitorar a evolução de pacientes ${ }^{5}$.

Atualmente, existem várias medidas para a avaliação funcional dos MMSS para indivíduos pós-AVE ${ }^{5}$. $\mathrm{O}$ questionário ABILHAND é uma delas e mede um construto específico, a habilidade manual, definida como a habilidade de gerir atividades diárias, que requerem o uso dos MMSS, independentemente das estratégias envolvidas ${ }^{6,7}$. A primeira versão do ABILHAND para pacientes com artrite reumatóide continha 56 itens, envolvendo tanto atividades unimanuais, quanto bimanuais ${ }^{6}$. Já a segunda versão, específica para pacientes pós-AVE, contém 23 itens, sendo todos sobre atividades bimanuais ${ }^{7}$. $\mathrm{O}$ estudo de validação do ABILHAND para indivíduos pós-AVE demonstrou que as atividades unimanuais foram muito fáceis, já que eles poderiam realizá-las utilizando apenas a mão não parética? ${ }^{7}$.

O referencial teórico do ABILHAND se baseia na Classificação Internacional de Funcionalidade, Incapacidade e Saúde (CIF) ${ }^{8}$. O questionário contempla diversos domínios do componente Atividade e Participação, incluindo áreas da vida como mobilidade, cuidado pessoal e vida doméstica9 ${ }^{9}$ O componente Atividade e Participação da CIF apresenta dois qualificadores: capacidade e desempenho. Capacidade é a habilidade máxima de um indivíduo para executar uma tarefa em um ambiente padronizado, enquanto desempenho descreve o que o indivíduo faz no seu ambiente de vida real ${ }^{9}$. O ABILHAND se enquadra como uma medida de desempenho, mais especificamente como autopercepção do desempenho, pois mede a habilidade manual a partir da percepção do indivíduo sobre seu desempenho ao realizar atividades manuais durante sua vida diária ${ }^{5}$.
O ABILHAND foi construído baseado no modelo Rasch de mensuração, que permite a transformação de escores ordinais em medida lineares ${ }^{7}$. Assim, no ABILHAND, a medida de habilidade manual é obtida pelo posicionamento dos indivíduos ao longo de uma escala unidimensional, com unidades iguais e metricamente definidas pelo nível de dificuldade das atividades ${ }^{6,7}$. Por outro lado, escores ordinais, utilizados em diversos questionários, dependem de contagens de unidades potencialmente desiguais e, por isso, representam um problema, quando utilizados diretamente como medida ${ }^{10}$. A vantagem das medidas lineares é que elas podem oferecer resultados cientificamente mais robustos e clinicamente mais significativos do que medidas ordinais, ao permitir inferências corretas a partir de comparações diretas inter ou intraindivíduos ${ }^{11}$.

O ABILHAND, específico para indivíduos pósAVE, foi utilizado em estudos prévios ${ }^{12,13}$ e apresentou adequada validade de construto ${ }^{7}$ e confiabilidade testereteste $^{14}$. Além disso, pode ser recomendado para uso clínico, por pontuar nove em 10 na escala de utilidade clínica de Tyson e Connell, que avalia o tempo de aplicação, custo, necessidade de equipamento, treinamento especial e portabilidade ${ }^{15}$.

Portanto, o ABILHAND é uma medida potencialmente útil para ser utilizada no Brasil. Porém, para sua aplicação na população brasileira, é necessária sua adaptação transcultural. Tal processo requer uma metodologia específica, a fim de alcançar equivalência entre a fonte original e a língua-alvo ${ }^{16,17}$. Além disso, a adaptação transcultural apresenta vantagens, pois, além de disponibilizar uma medida, que pode ser aplicada em diversos contextos culturais e utilizada em estudos internacionais, os custos financeiros e consumo de tempo são menores, quando comparados aos de desenvolvimento de novas medidas ${ }^{16,17}$. Assim, o objetivo do presente estudo foi realizar a adaptação transcultural da versão em inglês do ABILHAND específico para indivíduos pós-AVE, para possibilitar o seu uso no Brasil.

\section{MÉTODOS}

Este estudo é parte de um projeto maior, intitulado Preditores da Restrição na Participação Social de Hemiparéticos Crônicos, que foi aprovado pelo Comitê de Ética em Pesquisa da Universidade Federal de Minas Gerais sob o $\mathrm{n}^{\circ}$ CAAE 06609312.0.0000.5149. Os criadores do ABILHAND, Máximo Penta, Jean-Louis Thonnard e Luigi Tesio, autorizaram a sua adaptação transcultural e durante a realização deste estudo, receberam informações 
sobre as etapas desenvolvidas. O processo de adaptação transcultural teve início em agosto de 2012 e finalização em março de 2013.

\section{Aplicação e interpretação do ABILHAND}

O ABILHAND, específico para indivíduos pós-AVE, contém uma escala de pontuação de três níveis: impossível, difícil ou fácil ${ }^{7}$. De acordo com as instruções de aplicação e interpretação ${ }^{18}$, o ABILHAND deve ser aplicado sob a forma de entrevista, na qual o indivíduo é solicitado a avaliar a sua facilidade ou dificuldade ao desempenhar cada atividade, quando as mesmas são realizadas sem ajuda, independentemente do(s) membro(s) superior(es) e estratégias utilizadas. Atividades nunca realizadas ou não tentadas nos últimos três meses não são pontuadas.

As respostas devem ser submetidas à uma análise online gratuita no site www.rehab-scales.org ${ }^{18}$, que converte os escores ordinais em medida lineares, baseada no modelo Rasch de mensuração. Após a submissão das respostas, é gerado um relatório de avaliação, no qual a habilidade manual do indivíduo e seu intervalo de confiança de $95 \%$ estão localizados em uma escala linear de habilidade manual, expressa em logits. Nessa escala, o zero é convencionalmente definido como a dificuldade média dos itens e medidas positivas e negativas indicam uma habilidade maior e menor que essa média, respectivamente ${ }^{8}$.

O relatório de avaliação também apresenta um mapa de itens, no qual as atividades estão listadas em ordem decrescente de dificuldade. Nesse mapa, o escore mais provável $(0=$ =Impossível; $1=$ Difícil; $2=$ Fácil $)$ para cada item é uma função da habilidade manual do indivíduo e da dificuldade do item. Dessa forma, "martelar um prego" é o item mais difícil, porque requer uma maior habilidade para ser realizado facilmente e "lavar as mãos" é o mais fácil, porque requer uma menor habilidade para ser realizado facilmente ${ }^{18}$.

No relatório de avaliação, a resposta do indivíduo em cada item é circulada e nenhuma marcação é feita para as respostas não pontuadas (ponto de interrogação). Para facilitar a interpretação dos resultados, além da representação gráfica, o relatório também contém o valor exato da medida de habilidade manual, fornecido em "Patient measure". Essa é a medida que deve ser utilizada como escore final.

\section{Procedimentos}

A adaptação transcultural do ABILHAND foi realizada conforme as recomendações de Beaton et al. ${ }^{16} \mathrm{e}$ Wild et al. ${ }^{19}$, sendo organizada em cinco estágios (Figura 1).

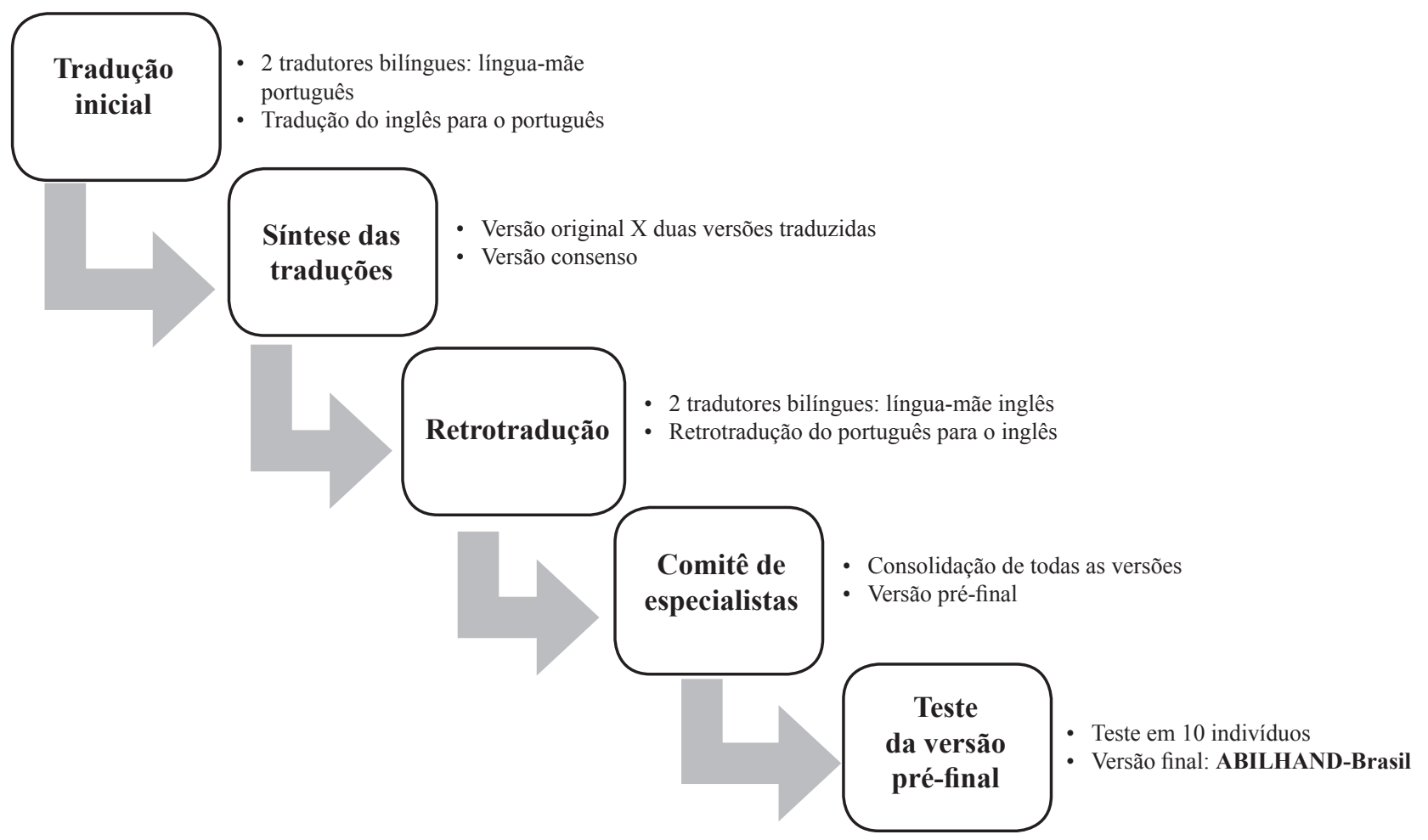

Figura 1 - Estágios do processo de adaptação transcultural do ABILHAND 
1. Tradução inicial: a tradução do ABILHAND do inglês para o português-Brasil foi realizada de forma independente, por dois tradutores bilíngües, cuja língua-mãe era o português, sendo que um dos tradutores estava ciente dos conceitos examinados pelo questionário. Assim, sua tradução poderia oferecer uma equivalência mais confiável, a partir da perspectiva do questionário. O segundo não foi informado sobre os conceitos do questionário, pois o objetivo era que esse tradutor produzisse uma versão com a linguagem utilizada pela população de interesse, o que poderia evidenciar significados ambíguos no questionário original.

2. Síntese das traduções: uma equipe composta por pesquisadores envolvidos no projeto, com experiência em adaptações de instrumentos e reabilitação de indivíduos pós-AVE, elaborou uma síntese das traduções, por meio da comparação da versão original com as duas versões traduzidas. A versão unificada resultante desse processo foi denominada versão-consenso.

3. Retrotradução: a retrotradução foi realizada a partir da versão-consenso, de forma independente por outros dois tradutores bilíngües, cuja línguamãe era o inglês. Eles não tiveram acesso ao material original, para ser possível verificar de forma válida se a versão traduzida refletiu o mesmo conteúdo da versão original.

4. Comitê de especialistas: O comitê de especialistas foi composto pelos pesquisadores envolvidos no projeto, sendo três fisioterapeutas, uma terapeuta ocupacional e dois tradutores (um envolvido na etapa de tradução e outro na de retrotradução). O papel desse comitê foi consolidar todas as versões do questionário e desenvolver uma versão pré-final para ser testada na população de interesse.

5. Teste da versão pré-final: $\mathrm{O}$ teste da versão pré-final foi realizado com indivíduos pós-AVE, população alvo do questionário. Uma amostra de indivíduos da comunidade com história de AVE foi recrutada por meio de contatos com profissionais da rede de reabilitação e listas de projetos de pesquisa anteriores. Os critérios de inclusão foram: diagnóstico de AVE há mais de seis meses; idade $\geq 20$ anos e hemiplegia/hemiparesia unilateral de membros superiores, caracterizada pelo aumento de tônus dos músculos flexores de $\operatorname{cotovelo}^{20} \mathrm{e} /$ ou pela diferença superior a $10 \%$ entre as medidas de preensão manual entre o membro parético e o não parético ${ }^{21}$. Foram excluídos indivíduos com déficits cognitivos, avaliados pelo Mini-Exame do Estado Mental, para o qual 13 foi o ponto de corte para analfabetos, 18 para baixa e média escolaridade e 26 para alta escolaridade ${ }^{22}$, com afasia de expressão, que impossibilitasse o indivíduo de responder as perguntas verbalmente e com outras condições neuromusculoesqueléticas incapacitantes. Após a assinatura do termo de consentimento livre e esclarecido e de uma avaliação inicial para verificação dos critérios de elegibilidade e coleta de dados sociodemográficos, cada entrevistado respondeu ao questionário e foi solicitado a descrever como ele interpretou cada item. As entrevistas foram realizadas pela primeira autora do estudo.

\section{RESULTADOS E DISCUSSÃO}

O ABILHAND, específico para indivíduos pós-AVE, foi adaptado para uso no Brasil, seguindo procedimentos padronizados, a fim de se garantir a equivalência semântica, idiomática, cultural e conceitual da versão adaptada, em relação à origina ${ }^{16}$. A comparação entre a versão original e a retrotraduzida mostrou que a maioria dos itens se manteve inalterada ou com poucas alterações, o que demonstra a boa equivalência entre as versões. Entretanto, discretas divergências no uso de expressões foram observadas nos itens, em que verbos no gerúndio estavam presentes. Sendo assim, esses itens foram alterados para o infinitivo, para adequá-los às regras gramaticais da língua portuguesa. Além disso, no item 12 "Tearing open a pack of chips" foi acrescida a expressão "rasgando a embalagem", para melhorar a equivalência conceitual e ficar clara a forma de execução requerida nesse item. Essas modificações foram realizadas pelo comitê de especialistas durante a elaboração da versão pré-final. Na Tabela 1, são apresentados os itens pareados, descritos em inglês e em português, após alterações do comitê de especialistas. Por fim, este comitê também analisou a equivalência cultural, concluindo que as atividades descritas eram condizentes com o contexto cultural da sociedade brasileira. 
Tabela 1 - Itens do ABILHAND descritos em inglês e em português

\begin{tabular}{|c|c|c|}
\hline & Versão em Inglês & Versão em Português - Brasil \\
\hline 1. & Hammering a nail & Martelar um prego \\
\hline 2. & Threading a needle & Enfiar linha na agulha \\
\hline 3. & Peeling potatoes with a knife & Descascar batatas com uma faca \\
\hline 4. & Cutting one's nails & Cortar todas as unhas das mãos \\
\hline 5. & Wrapping up gifts & Embrulhar presentes \\
\hline 6. & Filing one's nails & Lixar todas as unhas das mãos \\
\hline 7. & Cutting meat & Picar carne \\
\hline 8. & Peeling onions & Descascar cebolas \\
\hline 9. & Shelling hazel nuts & Quebrar castanhas/nozes \\
\hline 10. & Opening a screw-topped jar & Abrir um pote com tampa de rosca \\
\hline 11. & Fastening the zipper of a jacket & Fechar o zíper de uma jaqueta \\
\hline 12. & Tearing open a pack of chips & Abrir um pacote de salgadinhos, rasgando a embalagem \\
\hline 13. & Buttoning up a shirt & Abotoar uma camisa \\
\hline 14. & Sharpening a pencil & Apontar um lápis \\
\hline 15. & Spreading butter on a slice of bread & Passar manteiga no pão \\
\hline 16. & Fastening a snap (jacket, bag, ...) & Abotoar um botão de pressão (jaqueta, bolsa,...) \\
\hline 17. & Buttoning up trousers & Abotoar as calças \\
\hline 18. & Taking the cap off a bottle & Desenroscar a tampa de uma garrafa \\
\hline 19. & Opening mail & Abrir um envelope \\
\hline 20. & Squeezing toothpaste on a toothbrush & Colocar pasta de dente na escova \\
\hline 21. & Pulling up the zipper of trousers & Fechar o zíper das calças \\
\hline 22. & Unwrapping a chocolate bar & Abrir uma barra de chocolate \\
\hline 23. & Washing one's hands & Lavar as duas mãos \\
\hline
\end{tabular}


Para o teste da versão pré-final, foram recrutados 10 indivíduos, dos quais oito eram homens, com média de idade de $59 \pm 9$ anos. Dois tinham até quatro anos de escolaridade, três até oito anos, três até 11 e dois tinham no mínimo 15 anos. Todos os participantes do estudo foram capazes de responder a todos os itens do ABILHAND-Brasil em um tempo máximo de aplicação de 10 minutos. Não foram reportadas dificuldades de compreensão durante a aplicação, o que indica a equivalência conceitual entre as versões do questionário, assegurando a equivalência à versão original em uma situação aplicada ${ }^{16}$.
Como em trabalhos prévios ${ }^{23,24}$, resultados satisfatórios foram obtidos no teste da versão pré-final aplicada em 10 indivíduos, uma vez que não houve nenhum problema quanto à redação e clareza dos itens ou ao objetivo do questionário. Este fato pode ser decorrente da seleção e redação dos itens, que abrangem atividades cotidianas descritas por meio de itens de estrutura simples e objetiva, o que facilita a compreensão dos mesmos. Desta forma, foi concluída a versão final do ABILHAND, denominada ABILHAND-Brasil (Figura 2).

ABILHAND - Medida de Habilidade Manual

Versão em Português-Brasil

Paciente:

Data:

\begin{tabular}{|c|c|c|c|c|c|}
\hline & $\begin{array}{l}\text { Quâo DIFlCEIS } \\
\text { sắo as seguintes atividades? }\end{array}$ & Impossivel & Dificil & Fácil & ? \\
\hline 1. & Fechar o ziper das calças & & & & \\
\hline 2. & Descascar cebolas & & & & \\
\hline 3. & Apontar um lápis & & & & \\
\hline 4. & Desenroscar a tampa de uma garrafa & & & & \\
\hline 5. & Lixar todas as unhas das mãos & & & & \\
\hline 6. & Descascar batatas com uma faca & & & & \\
\hline 7. & Abotoar as calçars & & & & \\
\hline 8. & Abrir um pote com tampa de rosca & & & & \\
\hline 9. & Cortar todas as unhas das mäos & & & & \\
\hline 10. & $\begin{array}{l}\text { Abrir um pacote de salgadinhos, rasgando a } \\
\text { embalagem }\end{array}$ & & & & \\
\hline 11. & Abrir uma barra de chocolate & & & & \\
\hline 12. & Martelar um prego & & & & \\
\hline 13. & Passar manteiga no pão & & & & \\
\hline 14. & Lavar as duas mãos & & & & \\
\hline 15. & Abotoar uma camisa & & & & \\
\hline 16. & Enfiar linha na agulha & & & & \\
\hline 17. & Picar carne & & & & \\
\hline 18. & Embrulhar presentes & & & & \\
\hline 19. & Fechar o ziper de uma jaqueta & & & & \\
\hline 20. & $\begin{array}{l}\text { Abotoar um botão de pressão (jaqueta, } \\
\text { bolsa,...) }\end{array}$ & & & & \\
\hline 21. & Quebrar castanhas/nozes & & & & \\
\hline 22. & Abrir um envelope & & & & \\
\hline 23. & Colocar pasta de dente na escova & & & & \\
\hline
\end{tabular}

Figura 2 - ABILHAND-Brasil 
A metodologia proposta por Beaton et al. ${ }^{16} \mathrm{e}$ Wild et al ${ }^{19}$ assegurou a validade de face e de conteúdo do ABILHAND-Brasil, sendo esta a primeira etapa do processo de validação de um instrumento. Após o processo de adaptação transcultural, outras propriedades de medida, tais como validade de construto e confiabilidade, devem ser investigadas, para determinar se o questionário é adequado para a aplicação pretendida. A avaliação das propriedades de medida do ABILHAND-Brasil foi realizada por Basílio et al. ${ }^{25}$. Nesse estudo, os resultados da análise Rasch mostraram que o ABILHAND-Brasil apresentou uma escala de pontuação apropriada, confiabilidade e validade de construto adequadas ${ }^{25}$. Além disso, o ABILHANDBrasil também apresentou validade transcultural, pois observou-se que a calibração dos itens do ABILHANDOriginal e do ABILHAND-Brasil forneceram medidas iguais de habilidade manual ${ }^{25}$.

\section{CONSIDERAÇÕES FINAIS}

O questionário ABILHAND demonstrou ser uma boa opção, para se avaliar o desempenho de indivíduos pós-AVE em atividades bimanuais. Medidas de desempenho são importantes para planejar e avaliar intervenções direcionadas para melhorar a funcionalidade nos contextos de vida real dos indivíduos. No entanto, a avaliação de indivíduos no ambiente real, muitas vezes, não é viável dentro de contextos clínicos. Assim, as medidas de autopercepção fornecidas pelo ABILHAND apresentam grande utilidade no processo de reabilitação.

Os resultados desse estudo viabilizam o uso do ABILHAND no Brasil. O ABILHAND-Brasil, que inclui o manual de aplicação e as 10 ordens aleatórias, já está disponível gratuitamente no site www.rehab-scales.org ${ }^{18}$. O questionário é rápido e pode ser aplicado facilmente em indivíduos com diferentes graus de escolaridade e níveis socioeconômicos. Como o ABILHAND é um questionário, recomenda-se que o avaliador enfatize as instruções de aplicação, para diminuir a possibilidade de respostas aleatórias por parte do respondente. Além disso, por se tratar de uma medida construída de acordo com o modelo Rasch de mensuração, a utilização de escores brutos não é recomendável, sendo indispensável a submissão das respostas à análise on-line, também disponível gratuitamente no site www.rehab-scales. $\operatorname{org}^{18}$, para obtenção do escore linear de habilidade manual proveniente da aplicação do ABILHAND-Brasil.

Autoria e indicação de responsabilidade: M.L. Basílio, I. Faria-Fortini e F.S.N. Assumpção contribuíram com a concepção, planejamento, organização e desenvolvimento do projeto; participaram de todo o seu desenvolvimento, desde a coleta dos dados, interpretação dos resultados até a elaboração do manuscrito, revisão crítica do seu conteúdo e aprovação da sua versão final. A.C. Carvalho contribuiu com o desenvolvimento do projeto, participando da coleta dos dados, interpretação dos resultados, elaboração do manuscrito e revisão crítica do seu conteúdo e aprovação da sua versão final. L.F. Teixeira-Salmela colaborou com a concepção, planejamento e desenvolvimento do projeto, participando da interpretação dos resultados, elaboração do manuscrito e revisão crítica do seu conteúdo e aprovação da sua versão final.

\section{REFERÊNCIAS}

1. Brasil. Ministério da Saúde. Secretaria de Atenção à Saúde. Departamento de Ações Programáticas Estratégicas. Diretrizes de atenção à reabilitação da pessoa com acidente vascular cerebral. Brasília: Ministério da Saúde; 2013. Disponível em: http://bvsms.saude.gov.br/bvs/publicacoes/diretrizes atencao_reabilitacao_acidente_vascular_cerebral.pdf.

2. Carod-Artal FJ. Determining quality of life in stroke survivors. Expert Rev Pharmacoecon Outcomes Res. 2012;12(2):199-211. doi: 10.1586/erp.11.104.

3. Pollock A, St George B, Fenton M, Firkins L. Top 10 research priorities relating to life after stroke - consensus from stroke survivors, caregivers, and health professionals. Int J Stroke. 2014;9(3):313-20. doi: 10.1111/j.1747-4949.2012.00942.x.
4. Harris JE, Eng JJ. Paretic upper-limb strength best explains arm activity in people with stroke. Phys Ther. 2007;87:8897. doi: $10.2522 / \mathrm{ptj} .20060065$.

5. Lemmens RJ, Timmermans AA, Janssen-Potten YJ, Smeets RJ, Seelen HA. Valid and reliable instruments for arm-hand assessment at ICF activity level in persons with hemiplegia: a systematic review. BMC Neurol. 2012;12:21. doi: 10.1186/1471-2377-12-21.

6. Penta M, Thonnard JL, Tesio L. ABILHAND: a Raschbuilt measure of manual ability. Arch Phys Med Rehabil. 1998;79(9):1038-42. doi: http://dx.doi.org/10.1016/S00039993(98)90167-8. 
7. Penta M, Tesio L, Arnould C, Zancan A, Thonnard JL. The ABILHAND Questionnaire as a measure of manual ability in chronic stroke patients: Rasch-based validation and relationship to upper limb impairment. Stroke. 2001;32:1627-34. doi: 10.1161/01.STR.32.7.1627.

8. Penta M. ABILHAND: a Rasch-built measure of manual ability in upper limb impaired patients [Thesis]. Louvain: Faculté de Médicine, Université Catholique de Louvain; 2001 [cited 2016 July 22]. Available from: http://www. rehab-scales.org/abilhand-downloads.html\#thesis.

9. Organização Mundial de Saúde, Organização Panamericana de Saúde. CIF - Classificação Internacional de Funcionalidade, Incapacidade e Saúde. São Paulo: Edusp; 2003.

10. Wright BD, Linacre JM. Observations are always ordinal; measurements, however, must be interval. Arch Phys Med Rehabil. 1989;70(12):857-60.

11. Grimby G, Tennant A, Tesio L. The use of raw scores from ordinal scales: time to end malpractice? J Rehabil Med. 2012;44:97-8. doi: 10.2340/16501977-0938.

12. Alt Murphy M, Persson HC, Danielsson A, Broeren J, Lundgren-Nilsson A, Sunnerhagen KS. SALGOT-Stroke Arm Longitudinal study at the University of Gothenburg, prospective cohort study protocol. BMC Neurol. 2011;11:56. doi: 10.1186/1471-2377-11-56.

13. Liao WW, Wu CY, Hsieh YW, Lin KC, Chang WY. Effects of robot-assisted upper limb rehabilitation on daily function and real-world arm activity in patients with chronic stroke: a randomized controlled trial. Clin Rehabil. 2012;26(2):11120. doi: 10.1177/0269215511416383.

14. Ekstrand E, Lindgren I, Lexell J, Brogårdh C. Test-retest reliability of the ABILHAND Questionnaire in persons with chronic stroke. PM R. 2014;6:324-31. doi: 10.1016/j. pmrj.2013.09.015.

15. Connell LA, Tyson SF. Clinical reality of measuring upperlimb ability in neurologic conditions: a systematic review. Arch Phys Med Rehabil. 2012;93:221-8. doi: 10.1016/j. apmr.2011.09.015.

16. Beaton DE, Bombardier C, Guillemin F, Ferraz MB. Guidelines for the process of cross-cultural adaptation of self-report measures. Spine. 2000;25(24):3186-91.

17. Costner WJ, Mancini MC. Recomendações para a tradução e adaptação transcultural de instrumentos para a pesquisa e a prática em terapia ocupacional. Rev Ter Ocup Univ São
Paulo. 2015;26(1):50-7. doi: http://dx.doi.org/10.11606/ issn.2238-6149.v26i1p50-57.

18. Université catholique de Louvain. Rehab-scales.org [Internet]. 2007 [cited 2016 July 22]. Available from: http:// www.rehab-scales.org/.

19. Wild D, Grove A, Martin M, Eremenco S, McElroy S, Verjee- Lorenz A, Erikson P; ISPOR Task Force for Translation and Cultural Adaptation. Principles of good practice for the translation and cultural adaptation process for patient - reported outcomes (PRO) measures: report of the ISPOR Task Force for Translation and Cultural Adaptation. Value Health. 2005;8(2):94-104. doi: 10.1111/ j.1524- 4733.2005.04054.x.

20. Gregson JM, Leathley M, Moore AP, Sharma AK, Smith TL, Watkins CL. Reliability of the Tone Assessment Scale and the modified Ashworth Scale as clinical tools for assessing poststroke spasticity. Arch Phys Med Rehabil. 1999;80:1013-6. doi: http://dx.doi.org/10.1016/S00039993(99)90053-9.

21. Faria CDCM, Aguiar LT, Lara EM, Souza LAC, Martins JC, Teixeira-Salmela LF. Dynamometry for the assessment of grip, pinch, and trunk strength in subjects with chronic stroke: reliability and various sources of outcome values. Int J Phys Med Rehabil. 2013;1:168. doi:10.4172/23299096.1000168.

22. Bertolucci PH, Brucki SMD, Campacci SR, Juliano Y. O mini-exame do estado mental em uma população geral: impacto da escolaridade. Arq Neuro-Psiquiatr. 1994;52(1):1-7. doi: http://dx.doi.org/10.1590/S0004282X1994000100001.

23. Assumpção FSN, Faria-Fortini I, Basílio ML, Magalhães LC, Carvalho AC, Teixeira-Salmela LF. Adaptação transcultural do LIFE-H 3.1: um instrumento de avaliação da participação social. Cad Saude Publica. 2016;32(6):pii:S0102 311X2016000604001. doi: 10.1590/0102-311X00061015.

24. Faria-Fortini I, Basílio ML, Assumpção FSN, TeixeiraSalmela LF. Adaptação transcultural e reprodutibilidade do Measure of the Quality of the Enviroment em indivíduos com hemiparesia. Rev Ter Ocup Univ São Paulo. 2016;27(1):4251. doi: http://dx.doi.org/10.11606/issn.2238-6149. v27i1p42-51.

25. Basílio ML, Faria-Fortini I, Magalhães LC, Assumpção FSN, Carvalho AC, Teixeira-Salmela LF. Cross-cultural validity of the Brazilian version of the ABILHAND questionnaire for chronic stroke individuals, based on Rasch analysis. J Rehabil Med. 2016;48(1):6-13. doi: 10.2340/16501977-2044. 